\title{
INTERNATIONAL BIBLIOGRAPHY
}

OF HISTORICAL SCIENCES 



\section{INTERNATIONAL BIBLIOGRAPHY OF HISTORICAL SCIENCES}

INTERNATIONALE BIBLIOGRAPHIE DER GESCHICHTSWISSENSCHAFTEN BIBLIOGRAFIA INTERNACIONAL DE CIENCIAS HISTORICAS BIBLIOGRAPHIE INTERNATIONALE DES SCIENCES HISTORIQUES BIBLIOGRAFIA INTERNAZIONALE DELLE SCIENZE STORICHE

\section{VOLUME XV $1940-1946$}

Edited by Massimo Mastrogregori

with the contribution of a number of scholars, under the auspices of the International Committee of Historical Sciences 
The IBOHS for the years 1978 to 1992 (Vol. 47 - 61) was edited by Michel François and Michael Keul for Vol. 47/48 (1978/1979) and Jean Glénisson and Michael Kéul for Vol. 49 - 61 (1980 - 1992) on behalf of the International Committee of Historical Sciences and was published by K. G. Saur Munich.

Bibliographic information published by Die Deutsche Bibliothek

Die Deutsche Bibliothek lists this publication in the Deutsche Nationalbibliografie; detailed bibliographic data is available in the Internet at http://dnb.ddb.de.

$@$

Printed on acid-free paper / Gedruckt auf säurefreiem Papier

(C) 2005 by K. G. Saur Verlag GmbH, München Printed in Germany

All Rights Strictly Reserved / Alle Rechte vorbehalten

No part of this publication may be reproduced, stored in a retrieval system, or transmitted in any form or by any means, electronic, mechanical, photocopying, recording, or otherwise, without permission in writing from the publisher / Jede Art der Vervielfältigung ohne Erlaubnis des Verlags ist unzulässig

Technical partner: Dr. Rainer Ostermann, München Managing partner and technical support: Ellediemme libri dal mondo, Roma Printed and Bound by Strauss GmbH, Mörlenbach 
General editor

Massimo MASTROGREGORI, Università di Roma 'La Sapienza'

Assistant editor

Carlo COLELLA, Roma

Advisory board

Maria Tereza AMADO, Universidad de Evora

Girolamo ARNALDI, Istituto storico italiano per il Medioevo, Roma

$\dagger$ Yuri BESSMERTNY, Institute of General History, Russian Academy of Sciences,

Moscow

† Wiesław BIEŃKOWSKI, Polska Akademia Nauk

László BIRÓ, Hungarian Academy of sciences, Budapest

Corinne BONNET, Academia Belgica, Rome

Luciano CANFORA, Università di Bari

Alejandro CATTARUZZA, University of Buenos Aires, Argentina

Anne EIDSFELDT, University of Oslo Library

Ilse FREDERIKSEN VÄHÄKYRÖ, Turku University Library, Finland

Jean GLÉNISSON, Comité International des Sciences Historiques, Paris

Kazuhiko KONDO, University of Tokyo

Mario MAZZA, Università di Roma 'La Sapienza'

Vilém PREČAN, Institute of Contemporary History, Prague

Matjaz REBOLJ, Ljubljana

Jacques REVEL, Ecole des Hautes Etudes en Sciences Sociales, Paris

$\uparrow$ Ruggiero ROMANO, Ecole des Hautes Etudes en Sciences Sociales, Paris

Gabrielle M. SPIEGEL, Johns Hopkins University, Baltimore

Martina STERCKEN, Universität Zürich

Natasa STERGAR, Ljubliana

Abdeljellil TEMIMI, Fondation Temimi, Tunis

Şerban TURCUŞ, Università “Babeş-Bolyai”, Cluj Napoca

Nenad VEKARIĆ, Dubrovnik

Bahaeddin YEDİYILDIZ, Hacettepe Universitesi, Ankara

\section{Contributing editors}

Kira E. AGEEVA, Institute of Archaeology of the Russian Academy of Sciences, Moscow (Russian historiography)

Vassili N. BABENKO, Russian Academy of Sciences, Moscow (Russian Historiography)

$\dagger$ Wiesław BIEŃKOWSKI, Polska Akademia Nauk (Polish historiography)

Vera BRENOVA, Institute of Contemporary History, Prague (Czech historiography)

Alejandro CATTARUZZA, University of Buenos Aires, Argentina (Latin American

historiography)

Carlo COLELLA (History by countries, History of international relations)

Laura DE GIORGI, Università di Venezia (Chinese historiography)

Francesca DEVESCOVI, Trieste (History of modern culture) 
Anne EIDSFELDT, University of Oslo Library, (Norvegian historiography) Ilse FREDERIKSEN VÄHÄKYRÖ, Turku University Library, Finland (Finnish historiography)

Anna GRUCA, Instytut Historii PAN. Pracownia Bibliografii Bieżącej (Polish historiography)

Alessandro GUERRA, Università di Roma 'La Sapienza' (Modern religious history) Timophey GUIMON, Institute of Universal History of the Russian Academy of Sciences, Moscow (Russian historiography)

Kazuhiko KONDO, University of Tokyo (Japanese historiography)

Mauro LENZI, Società Romana di Storia Patria, Roma (Palaeography, Diplomatics, History of the book, Medieval history)

Jean Marie MAILLEFER, Université Charles-De-Gaulle Lille 3 (Danish and Swedish historiography)

Massimo MASTROGREGORI, Università di Roma 'La Sapienza' (Auxiliary sciences, General works, Modern history)

Stjepan MATKOVIĆ, (Croatian historiography)

Massimo PONTESILLI, Roma (History of modern culture)

Matjaz REBOLJ, Ljubljana (Slovenian historiography)

Marco SANTUCCI, Università di Roma 'La Sapienza' (Ancient history)

Evgeny E. SAVITSKI, Institute of Universal History of the Russian Academy of Sciences, Moscow (Russian historiography)

Alžbeta SEDLIAKOVÁ, (Slovak historiography)

A. SLIVA, Rossijskaja Akademija Nauk, Moskva (Russian historiography)

Natale SPINETO, Università di Torino (History of religions)

Natasa STERGAR, Ljubliana (Slovenian historiography)

Paola STIRPE, Università di Roma 'La Sapienza' (Ancient history)

Şerban TURCUŞ, Università “Babeş-Bolyai”, Cluj Napoca, (Romanian historiography)

Michaela VALENTE, Università di Roma 'La Sapienza' (Modern religious history) Galina A. YANKOVSKAYA, Perm State University, Perm (Russian historiography)

Bahaeddin YEDIYILDIZ, Hacettepe Universitesi, Ankara (Historiography of Turkey)

\section{Consulting editors}

Maurice AYMARD, Maison des sciences de l'homme, Paris

Eric BRIAN, Centre Alexandre Koyré, Paris

Louis CHATELLIER, Université de Nancy II

Sten EBBESEN, University of Copenhagen

Carlo FRANCO, Università di Venezia

Olivier GUYOTJEANNIN, Ecole nationale des Chartes, Paris

Michel MORINEAU, Paris

Brian TIERNEY, Cornell University, Ithaca

Giusto TRAINA, Università di Lecce

Pietro VANNICELLI, Università di Urbino

André VAUCHEZ, Ecole Française de Rome 
Special Assistant editors

Daniele MASTROGREGORI, Roma

Carlo SCOGNAMIGLIO, Roma

Michele VOLLARO, Roma 
